\title{
Combustion Species Sensor for SCRAMJET Flight Instrumentation
}

\author{
Shin-Juh Chen ${ }^{*}$, Joel A. Silver ${ }^{\dagger}$, and David S. Bomse ${ }^{\ddagger}$ \\ Southwest Sciences, Inc., 1570 Pacheco Street, Suite E-11, Santa Fe, NM 87505 \\ and \\ Chadwick C. Rassmussen ${ }^{\S}$ and James F. Driscoll \\ The University of Michigan, Department of Aerospace Engineering, 1320 Beal Avenue, Ann Arbor, MI 48109
}

\begin{abstract}
An in-situ, non-intrusive, compact, and rugged sensor system is been developed for simultaneously monitoring multiple combustion species in scramjets during flight. The sensor is based on diode laser absorption spectroscopy with sensitivity enhanced by wavelength modulation. Simultaneous multi-species concentration measurements are achieved using modulation-frequency multiplexing with several lasers and a single two-color photodiode detector. Measurements of methane, water vapor, and oxygen concentrations, and temperatures were simultaneously measured in a laboratory diffusion flame and in a cavity flameholder with methane injection in a supersonic freestream. No channel cross-talk is observed between the visible and near-infrared layers of the detector and between the laser modulation frequencies.
\end{abstract}

\section{Introduction}

\section{A. Motivation}

The development of a scramjet engine to power aircraft to hypersonic speeds has occupied researchers for more than 50 years. Moving from laboratory research to test flight, as evidenced by the successful test flight of NASA's X-43A hypersonic aircraft, sensors are needed during flight to gather information about aerodynamics and combustion. The optimization of engine performance parameters such as combustion efficiency, thrust maximization, reduction in pollutants, and minimization of exhaust signatures (for stealth) are essential. Measurements of chemical species and temperature in the scramjet engine during ground and flight tests are critical for evaluating engine performance.

The application of a laser-based optical technique to probe chemical species in a laboratory combustion system has been demonstrated by several research groups [1-8]. A laser-based absorption method provides several advantages as compared to methods of probe extraction, laser-induced fluorescence, etc. However, designing a compact and rugged multi-species gas sensor system that is suitable for test flight has yet to be fully explored. The sensor system for monitoring the combustion section of a scramjet during flight must also be nonintrusive and lightweight. A flight-worthy hardware will require substantial reduction in the physical size of current systems, reduction in the number of lasers and detectors, utilization of innovative optics, implementation of compact and stand-alone electronics, and ruggedization of the instrument.

\section{B. Multiple Species Measurement Techniques}

Simultaneous or nearly simultaneous detection of multiple gases by diode laser spectroscopy usually requires a

* Senior Research Scientist, Southwest Sciences, Inc., Santa Fe, NM, Member

${ }^{\dagger}$ Executive Vice President, Southwest Sciences, Inc., Santa Fe, NM

₹ Vice President for Operations, Southwest Sciences, Inc., Santa Fe, NM

${ }^{\S}$ Graduate Student, Aerospace Engineering Department, The University of Michigan, Ann Arbor, MI, Member

" Professor, Aerospace Engineering Department, The University of Michigan, Ann Arbor, MI, Associate Fellow 
separate laser for each gas; only in rare, fortuitous cases does one laser exhibit a wavelength tuning range that spans useful absorption lines of more than one gas. The challenge for multiple gas sensing is to combine the light output from several lasers, direct the beams along the same optical path, and perform a separate absorbance measurement for each gas (laser) in a timely manner. Three separate techniques are described. These techniques include time-division multiplexing (TDM), wavelength-division multiplexing (WDM), and modulation-frequency multiplexing (MFM).

Time-division multiplexing technique can be implemented using an electro-mechanical fiber-optic switch to select one laser beam at a time [9]. However, the optical components within the switch can increase the size of unwanted etalons. Although high repetition rates $(1.5 \mathrm{kHz})$ are possible, the signal-to-noise ratios are usually adequate for high absorbances only. The recent availability of a multi-sectioned tunable diode laser (e.g. Intune Technologies) that can be programmed to switch wavelength scans among several key absorption lines can circumvent the need for an optical switch, thus providing a true TDM system with high sensitivity. Although there will be a time lag (order of milliseconds) between each species measurement, the electronics are greatly simplified. However, the tunning range of a multisectioned tunable diode laser, which is typically $35 \mathrm{~nm}$, inhibits the selection of absorption lines that are most suitable for a specific gas species of interest. Wavelength coverage is limited, but only a single detector is required for each probed location.

Wavelength-division multiplexing technique disperses the combined beams from several laser sources onto a grating. The dispersed beams are collected by several detectors; each detector having a specific wavelength coverage for the chemical species of interest. To disperse multiple laser beams with a grating $[3,5,10]$ one must add optical paths that differ from gas to gas. Such a configuration greatly increases the footprint of the system. Added paths can be problematic when detecting gases that are ubiquitous in air (e.g. water vapor, carbon dioxide, oxygen). Purging of these extraneous paths are often required. Gratings, because they are diffractive elements, add unwanted optical interference fringes (etalons) that can interfere with detection of weak absorbances (i.e. low concentrations). The recent availability of a fully fiberized grating system eliminates these extraneous paths (e.g. Zolo Technologies), but optical interferences will remain a major issue. In a distributed sensor system, a grating system along with its set of detectors is required for each probing location of interest.

Modulation-frequency multiplexing technique has been demonstrated by Oh [11] for the simultaneous detection of $\mathrm{CO}$ and $\mathrm{CO}_{2}$ using two near-infrared diode lasers and a single detector. Each laser current is modulated at a different frequency and lock-in amplifiers are used to process the corresponding wavelength modulation spectroscopy (WMS) signals. WMS provides high-sensitivity detection when compared to a direct absorption method. In the system designed by $\mathrm{Oh}$, the $\mathrm{CO}$ laser is modulated at $50 \mathrm{kHz}$ whereas the $\mathrm{CO}_{2}$ laser is modulated at $40 \mathrm{kHz}$. One can combine the output from the lasers by using a $2 \times 1$ fiber-optic beam combiner and inject it into a single-mode optical fiber. The resulting beam exits the fiber through a collimating graded-index lens, is directed into the measurement volume, and focuses onto an InGaAs photodiode. Output from the photodiode is applied to a pair of lock-in amplifiers set to operate at 2f; one is referred to the $50-\mathrm{kHz}$ modulation source and the other to the $40-\mathrm{kHz}$ source. That technique is used in this research to detect several laser wavelengths with a single two-color photodiode detector. In a distributed system, only one detector is needed for each probing location of interest. Inexpensive lock-in amplifier chips are required for each measurement location to process the individual modulated laser wavelengths of interest.

\section{Scope of This Research}

This paper focuses on demonstrating the feasibility of a sensor system based on modulation-frequency multiplexing to simultaneously measure multiple combustion species. Wavelength modulation spectroscopy is used to enhance the detection sensitivity of the chemical species. This method provides true simultaneous, multi-species, and high-sensitivity measurement capabilities in a compact and rugged footprint. A laser is used for each gas of interest. The lasers can be distributed feedback lasers (DFBs), vertical cavity surface-emitting lasers (VCSELs), multi-sectioned tunable diode lasers (MTDLs), etc. However, only a single two-color photodiode detector is used here. A conceptual view of the overall sensor system is illustrated in Fig. 1. Laser beams from several fiberized lasers are combined into a single beam using a fiber-optic beam combiner. The combined beam exits the combiner via another fiber-optic cable, and is injected into the measurement location. After traversing the probing region, the transmitted beam is collected onto a photodiode detector. As an alternative, the transmitted beam can be captured by another fiber optic cable with a collecting lens coupled at one end, and with a detector coupled at the other end. Simultaneous measurements of methane, water vapor, and oxygen concentration profiles, and temperature are demonstrated in a laboratory diffusion flame and a wall cavity, with methane injection, over a supersonic freestream. 


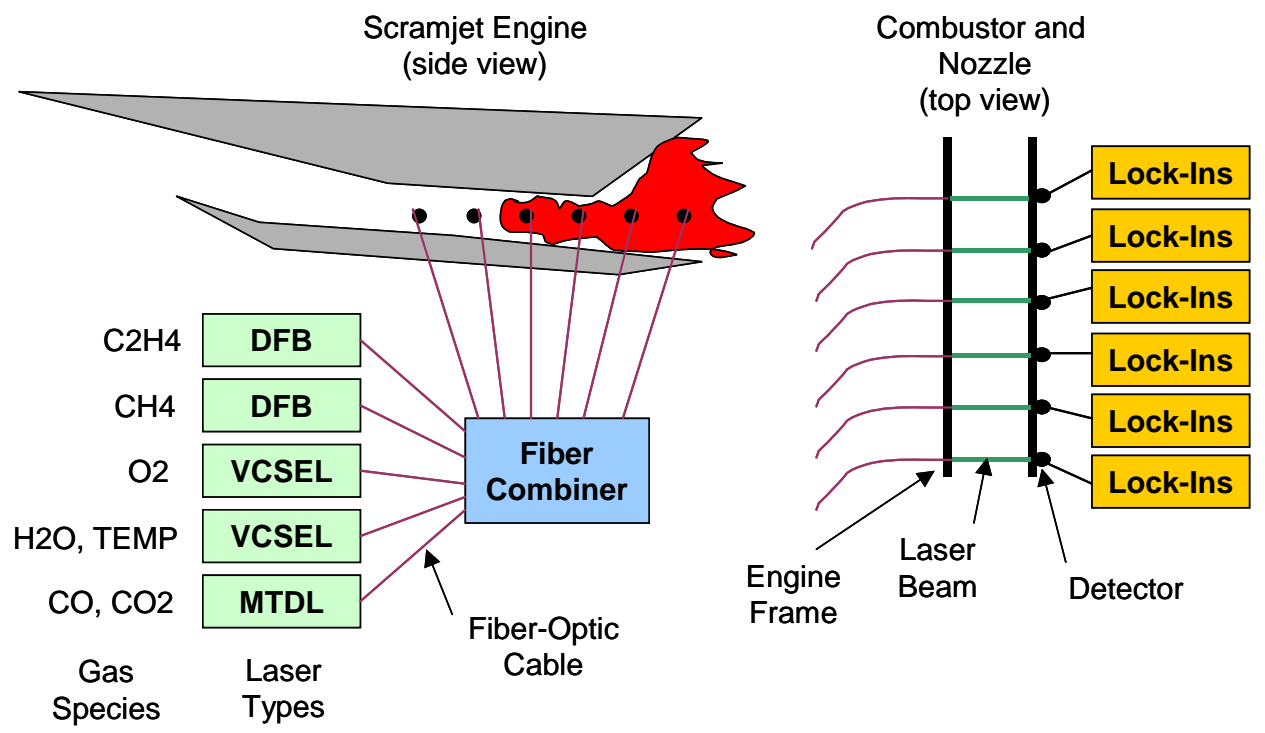

Figure 1. Schematic of a gas sensor system for a scramjet engine.

\section{Method and Apparatus}

\section{A. Diode Laser-Based Absorption Spectroscopy}

The measurement of chemical species concentrations by diode laser absorption is based on straightforward implementation of Beer's law,

$$
\frac{I}{I_{0}}=\exp (-\alpha), \quad \alpha=S(T) g(v) n \ell=\sigma(v) n \ell
$$

where $I_{0}$ is the light intensity incident on the gas sample, $I$ is the transmitted intensity and $\alpha$ the absorption coefficient. The absorption coefficient is comprised of the absorption line strength $S(T)$, the normalized line shape function $g(v)$, number density $n$ in $\mathrm{cm}^{-3}$ and path length $\ell$ in $\mathrm{cm}$. The line strength is a measure of how strongly light is absorbed and is a function of temperature but not pressure. The line shape function describes the wavelength dependence $(v)$ of the absorption and depends on both temperature and pressure.

A simple direct absorption spectrum using a diode laser is limited by low frequency noise, so that relative changes in the transmitted laser power of only $10^{-3}$ or greater can be detected. Techniques for achieving high sensitivity with diode lasers include wavelength modulation spectroscopy [12,13], frequency modulation spectroscopy [14], and "noise canceller” dual beam subtraction [15]. In all these techniques, the laser amplitude noise can be reduced to the theoretical shot noise limit, so that in practice, the noise in a spectral scan is dominated by coherent artifacts due to stray reflections in the optical path. These reflections interfere with the main laser beam ("etalon" effect) and produce ripples in the baseline.

Southwest Sciences has pioneered techniques for the measurement of weak optical absorbances [12,16-18]. High-sensitivity has been achieved by using wavelength modulation spectroscopy to shift the detection band from DC (where laser amplitude noise can obscure weak absorbances) to high frequencies where laser excess (1/f) noise is unimportant. Fractional absorption sensitivities near the shot-noise limit $\left(\sim 10^{-7}\right)$ have been achieved in laboratory experiments [17]. Field measurements using WMS routinely maintain minimum detection absorbances of better than $10^{-5}$ (1 $\mathrm{Hz}$ bandwidth) for extended (many month) operations [18-20].

To implement WMS, a small sinusoidal modulation at frequency $f$ is superimposed on the diode laser injection current. This current modulation

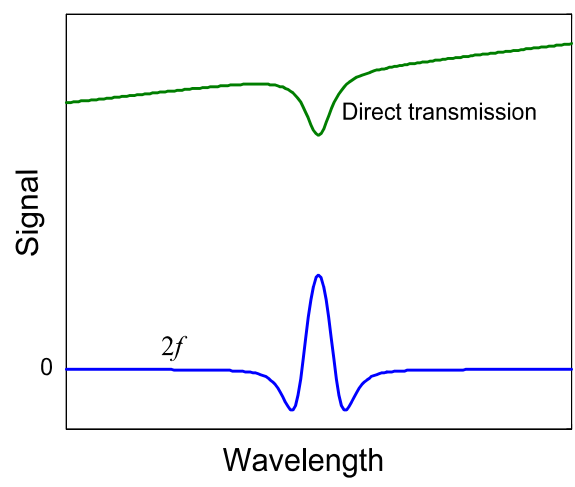

Figure 2. Direct transmission and corresponding $2 f$ signals. 
produces a modulation of the laser wavelength, since wavelength is tuned by changing the current. The amplitude of the current modulation is chosen so that the induced wavelength modulation is comparable to the width of the spectral feature under study. Absorption by the target gas converts the laser wavelength modulation to an amplitude modulation that induces ac components in the detector photo-current. Phase-sensitive electronics are then used to demodulate the detector photo-current at a selected harmonic, $n f$ (typically, $n=2$ ). Detection frequencies as low as $20 \mathrm{kHz}$ are often sufficient to bypass laser excess noise. The signal from this detection method appears as approximately the second derivative of the absorption feature with respect to wavelength (Fig. 2). Thus, $2 f$ signals have a zero background.

Flow temperatures are determined by measuring the ratio of absorption signals for two, or more, absorption lines. This ratio is proportional to the ratio of line strengths, which depends only on temperature and known molecular parameters. The line strength ratio for two absorption lines is

$$
\frac{S_{1}}{S_{2}}=\frac{S_{1}\left(T_{0}\right)}{S_{2}\left(T_{0}\right)} \exp \left[\frac{-h c \Delta E_{\text {rot }}}{k}\left(\frac{1}{T}-\frac{1}{T_{0}}\right)\right]
$$

where $T_{0}$ is a reference temperature (room temperature, for example) and $\Delta E_{\text {rot }}$ is the difference in lower state rotational energy for the two absorption lines. The use of two absorption lines has been explored by several researchers $[3,4,5,10]$. In this project, temperature measurements were conducted using peak ratios of two water absorption lines. This was accomplished by using a single $1854 \mathrm{~nm}$ water DFB laser that can scan two to three absorption lines which are close to each other $\left(<1 \mathrm{~cm}^{-1}\right.$ apart$)$.

The absorption lines for oxygen, methane, and water vapor are shown in Figs. 3-5. These lines are computed using SWSpectrum, a spectroscopic computation software

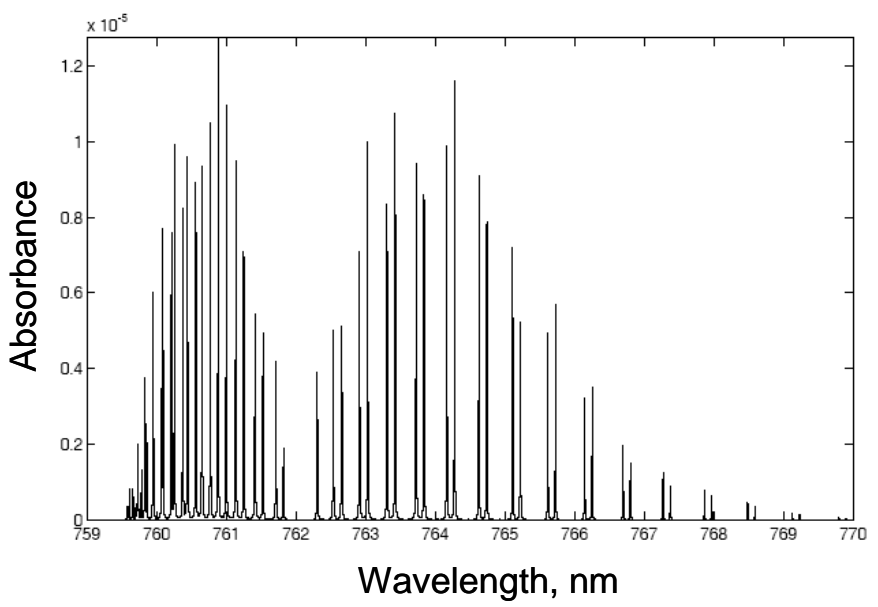

Figure 3. Oxygen absorption lines.

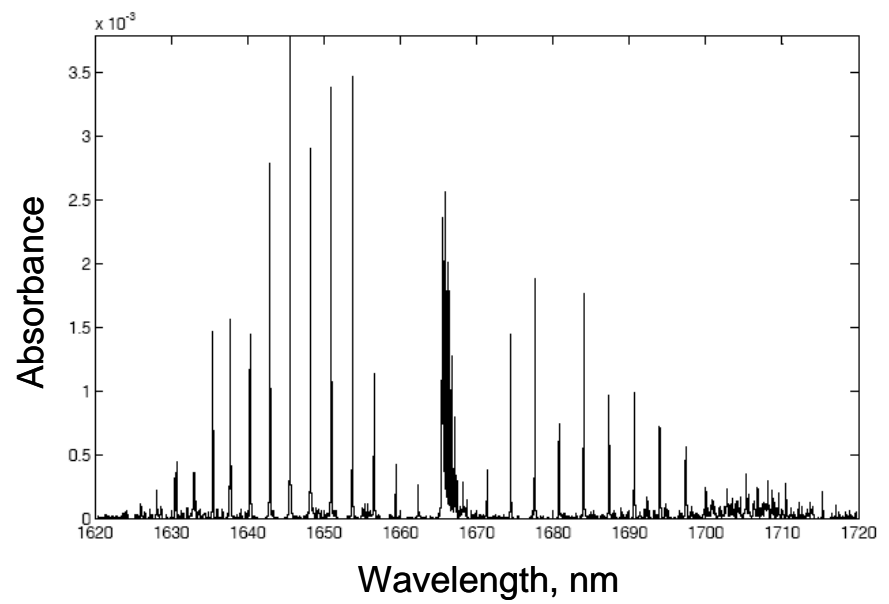

Figure 4. Methane absorption lines. developed by Southwest Sciences. The selected oxygen absorption lines span from 759 to $770 \mathrm{~nm}$. The absorbance is computed using 1 percent $\mathrm{O}_{2}$, and 99 percent $\mathrm{N}_{2}$, with an 1 -cm optical path. The $761 \mathrm{~nm}$ oxygen line is used in this project. The temperature and pressure are $300 \mathrm{~K}$ and 760 Torr for all computations. The methane absorption lines span from 1620 to $1720 \mathrm{~nm}$. The 1654 methane line is used. The water vapor lines span from 1800 to 1950 $\mathrm{nm}$. The water vapor lines between 1853.7 and 1854.2 are used for temperature and concentration measurements. The effect of temperature on water vapor absorption lines is demonstrated in Fig. 6. As temperature increases from 300 to $2000 \mathrm{~K}$ the absorption line is split into three distinct lines. The temperature is determined using peak ratios of lines labeled 1 and 3. The 1854 DFB laser is able to sweep these three lines over a single laser current scan.

\section{B. Two-Color Photodiode Detector}

This paper extends the modulation-frequency multiplexing technique by using lasers with wavelength spanning from 760 to $1900 \mathrm{~nm}$ and using a single two-color detector for measurements. The availability of two-color photodiode detectors 


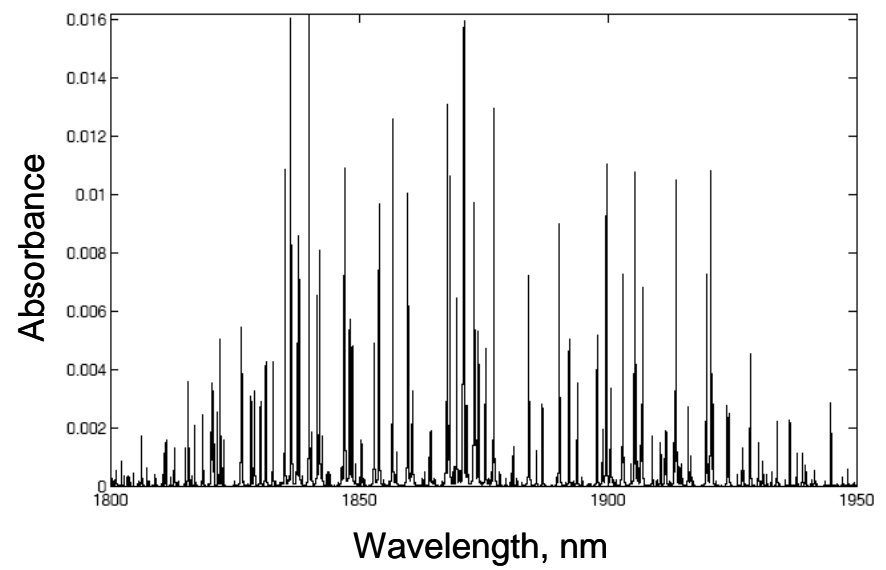

Figure 5. Water vapor absorption lines.

(Hamamatsu, K1713-08) with wide spectral response ranging from UV to IR allows the design of a common optical path when using separate visible and near-infrared lasers. The uncooled detector, in a metal TO-5 can, incorporates an infraredtransmitting $\mathrm{Si}$ photodiode mounted over an InGaAs PIN photodiode, along the same optical axis, each having a separate electrical output. The active area is $2.4 \mathrm{~mm} \times 2.4 \mathrm{~mm}$, and the operating temperature ranges from -40 to $+70{ }^{\circ} \mathrm{C}$. The storage temperature ranges from $-55+85^{\circ} \mathrm{C}$. The size of the detector, excluding the pins, is only $9.1 \mathrm{~mm}$ in diameter and $6.5 \mathrm{~mm}$ in height. The spectral response of the Si photodiode ranges from 0.25 to 1.1 microns with a peak around 0.95 microns. The InGaAs PIN photodiode has a spectral response ranging from 1.0 to 2.8 microns, with a plateau between 2.0 and 2.3 microns. The active area is $1 \mathrm{~mm} \times 1 \mathrm{~mm}$. The rise times are 200 and 3 ns for $\mathrm{Si}$ and InGaAs detector element, respectively. The Si detector element is for oxygen detection, and the InGaAs detector element is for methane and water vapor detection.
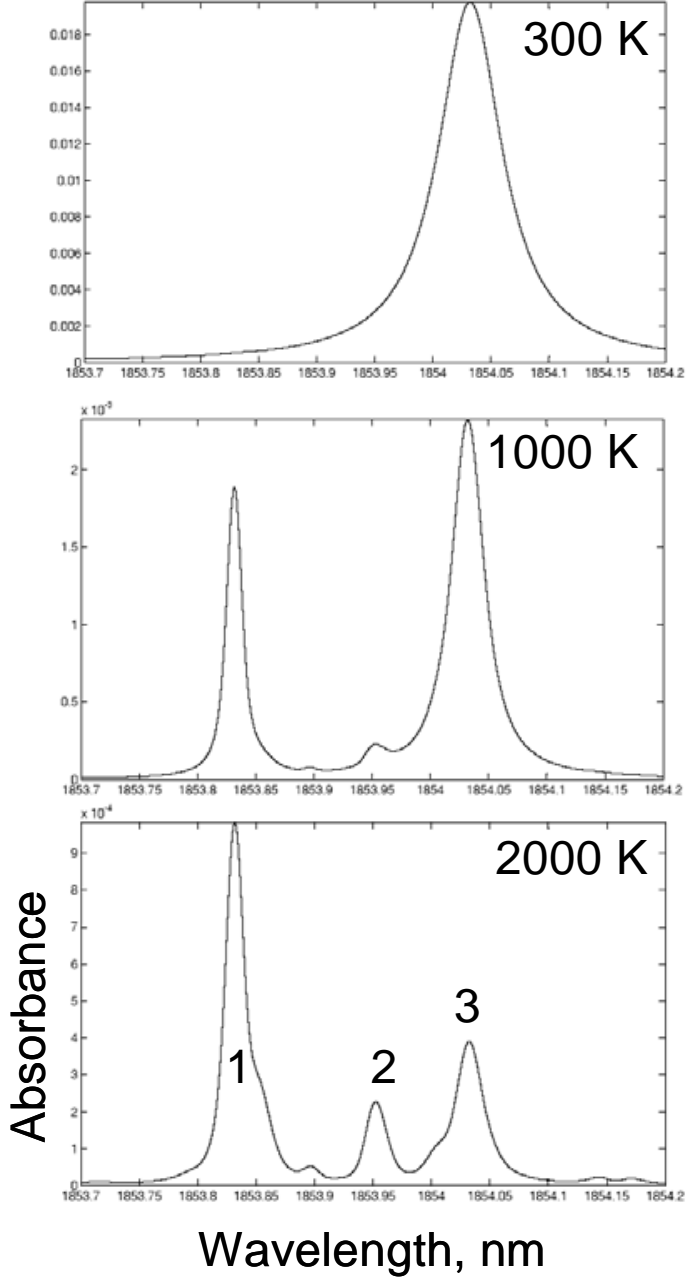

Figure 6. Temperature effect on water vapor absorption lines.

\section{Optical and Electronics Setup}

The laser system consists of three lasers to measure $\mathrm{O}_{2}, \mathrm{CH}_{4}, \mathrm{H}_{2} \mathrm{O}$, and temperature over the same optical path. Figure 7 shows the simple optical setup. The lasers include a $1654 \mathrm{~nm}$ DFB laser $\left(\mathrm{CH}_{4}\right), 1854 \mathrm{~nm} \mathrm{DFB}\left(\mathrm{H}_{2} \mathrm{O}\right)$, and a $760 \mathrm{~nm}$ VCSEL $\left(\mathrm{O}_{2}\right)$. The water laser beam passes thru a thin microscope slide and a high-pass filter to enter the measurement region. The methane laser beam reflects off the microscope slide and combines with the water laser beam. The oxygen laser beam reflects off the high-pass filter and combines with the other two beams. The combined three-laser beam enters the test section along the same optical path, and a two-color photodiode detector collects the beams exiting the test section. The detector is housed in a nitrogen-purged box. The three lasers are enclosed in a nitrogen-purged rectangular box as well. Nitrogen purging minimizes background concentrations of water vapor and oxygen inside the box. Fiberizing the lasers and combining the laser beams using a fiber-optic beam combiner will eliminate these extraneous paths.

Table-top electronics and special circuitry are used to control and modulate the lasers. Using custom digital signal processors, all the electronics can be reduced to a footprint no larger than a regular shoebox. Signals from the three lasers are simultaneously acquired using the two-color photodiode detector. Each laser is controlled by a current and temperature controller. Two output signals are collected from the detector. Each signal is fed to a current and voltage amplifier. Output from the current amplifier for the InGaAs detector element is fed to two lock-in amplifiers. Output from the current amplifier for the Si detector element is fed to a separate lock-in amplifier. Outputs from the current amplifiers are also fed to voltage amplifiers for recording the incident laser intensities. Optimally, two additional lock-in amplifiers would be used to monitor each laser intensity at 1 f simultaneously during the concentration measurements. 


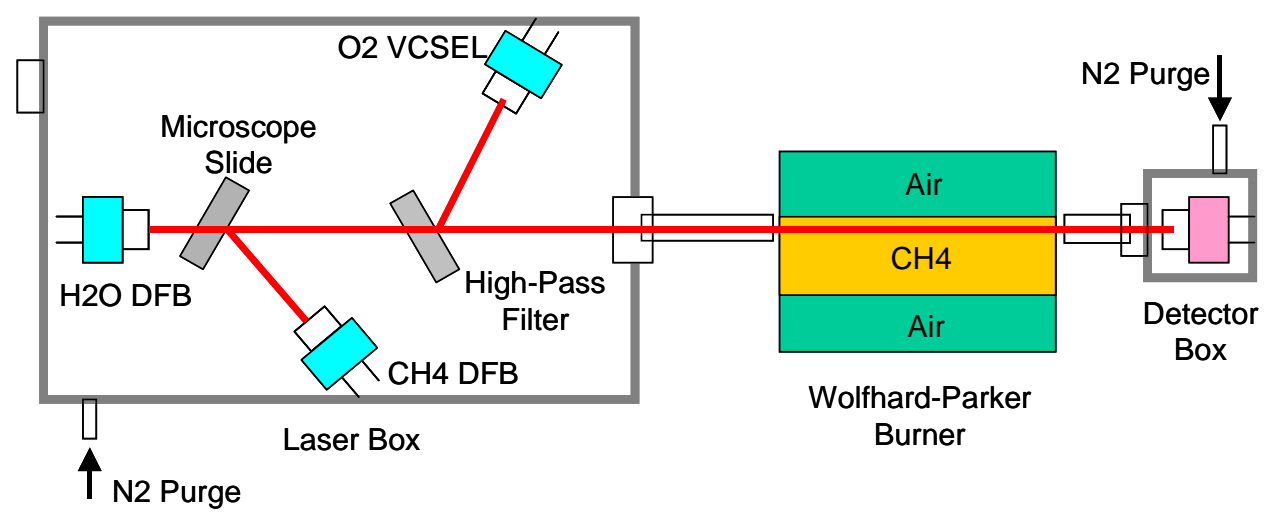

Figure 7. Schematic of optical setup.

A summing amplifier combines the laser ramp (at $10 \mathrm{~Hz}$ ), generated by the computer, and the modulation frequencies and amplitudes from the three lock-in amplifiers. The outputs from the summing amplifier is used to drive the laser current controllers. The incident laser intensities and WMS signals are acquired using a custom LabView program running from a laptop thru a NIDAQ data acquisition card. The card is able to accept 16 channels at 16 bit.

\section{Results}

\section{A. Channel Cross-Talk}

The Si detector element is mounted over the InGaAs element. Cross-talk is assessed between these two elements by measuring ambient oxygen and methane. A clear plastic bag containing pure methane is placed in front of the methane laser. Both the $\mathrm{O}_{2}$ and $\mathrm{CH}_{4}$ lasers are modulated at 40 $\mathrm{kHz}$. WMS signals of $\mathrm{O}_{2}$ are recorded while the $\mathrm{CH}_{4}$ laser is turned on, and vice versa. No cross-talk is observed between the silicon and InGaAs detector elements, as demonstrated in Fig. 8. The curves are offset for clarity. The WMS signals are acquired at $10 \mathrm{~Hz}$, and averaged over one second.

The InGaAs detector element is used to detect methane and water vapor WMS signals simultaneously. The $\mathrm{CH}_{4}$ laser is modulated at 35 $\mathrm{kHz}$, and the $\mathrm{H}_{2} \mathrm{O}$ laser at $40 \mathrm{kHz} ; 2 f$ detection is used for both. WMS signals of $\mathrm{CH}_{4}$ and $\mathrm{H}_{2} \mathrm{O}$ are shown in Fig. 9. No cross-talk is observed between the two recorded WMS signals. If the modulation

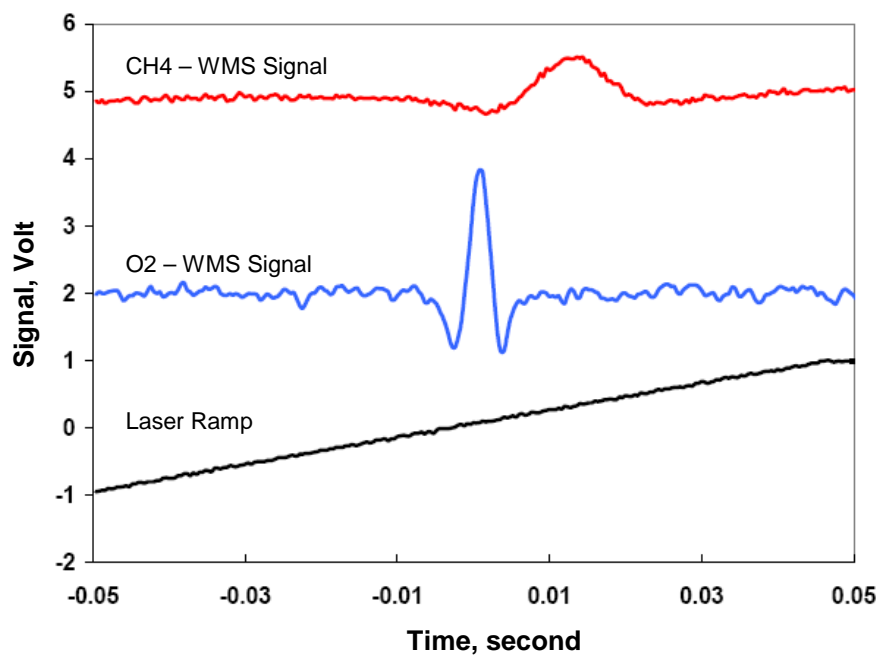

Figure 8. Methane and oxygen detection channels. frequencies are separated by $0.1 \mathrm{kHz}$ or less, then cross-talk is seen, as depicted in the top curve of Fig. 9. The minimum separation between the modulation frequencies is dependent on the lock-in amplifier frequency resolution. Additional lasers can be trivially added for detecting other chemical species by using different modulation frequencies. The InGaAs portion of the two-color photodiode detector has a spectral response between 1.1 and 2.8 microns. This wide spectral response allows species such as $\mathrm{CO}, \mathrm{CO}_{2}, \mathrm{NH}_{3}$, $\mathrm{C}_{2} \mathrm{H}_{4}$, etc to be detected as well.

Combining several laser wavelengths for simultaneous multi-species measurements using modulation-frequency multiplexing has very few restrictions. One, the modulation frequencies should not be harmonics of one another. Second, the modulation frequencies should be spaced appropriately within the frequency resolution of the lock-in amplifier been used. Third, chemical species that can be measured will depend on the availability of the laser at the specified wavelength, and the spectral response of the selected detector. 


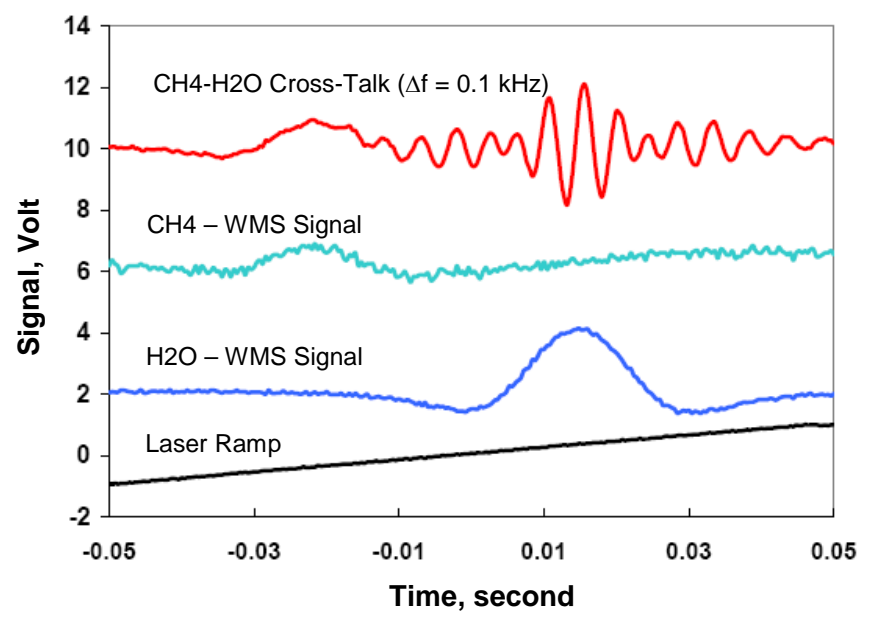

Figure 9. Methane and water vapor detection channels.

\section{B. Temperature and Chemical Species Profiles in a Diffusion Flame}

A diffusion flame generated by a WolfhardParker burner provides a simple test article to assess the capability of the multi-species sensor system. Oxygen, methane, and water vapor are measured simultaneously across a diffusion flame at $9 \mathrm{~mm}$ above the burner using the three-laser system. The burner consists of three slot nozzles with the fuel nozzle at the center. The center fuel nozzle is $4 \mathrm{~mm}$ $\times 40 \mathrm{~mm}$. An air nozzle is located on each side of the fuel nozzle. The air nozzle is $8 \mathrm{~mm} \times 40 \mathrm{~mm}$. Methane exits the central section of the burner at 11 $\mathrm{cm} / \mathrm{s}$, and air in the outer sections at $22 \mathrm{~cm} / \mathrm{s}$. The burner is mounted on a three-axis translation stage to allow species measurements at different flame heights and positions across the flame.

The laser and detector boxes were also nitrogen-purged to remove external optical paths. The background $\mathrm{O}_{2}$ and $\mathrm{H}_{2} \mathrm{O}$ were not completely removed due to the excessively long paths ( $>40 \mathrm{~cm}$ ) inside the laser box. Figure 10 shows a sample of the $2 f$ absorption spectra for the three chemical species at three different locations across the flame. $\mathrm{CH}_{4}$ peaks at center of the nozzle, while $\mathrm{O}_{2}$ peaks away from the flame. The $\mathrm{O}_{2}$ and $\mathrm{H}_{2} \mathrm{O}$ lasers were modulated at $41 \mathrm{kHz}$, and $\mathrm{CH}_{4}$ laser at $48.27 \mathrm{kHz}$. The incident laser intensity, $I_{o}$, for $\mathrm{CH}_{4}$ and $\mathrm{H}_{2} \mathrm{O}$ lasers can be easily collected with the two-color detector by $1 f$ detection; thus requiring two additional lock-ins. To simply the current electronics setup, the incident laser intensity, $I_{o}$, was collected for each individual laser prior to each experiment.

In Fig. 10, temperature profile across the diffusion flame is obtained using peak ratios of two water absorption lines, labeled 1 and 3 in Fig. 6. The computed temperature profile is compared to that obtained using a S-type thermocouple, including radiative corrections. Calibration of the water lines were not conducted to verify the spectroscopic parameters in the HITRAN/HITEMP database. The lack of calibration may have contributed to the discrepancies seen between the two temperature profiles. Nevertheless, the objective was to demonstrate the capability of the technique to simultaneously measure three chemical species and temperature in a flame.

\section{Methane Concentration Profiles in a Wall Cavity over a Supersonic Freestream}

Wall cavities have the capability to play the dual roles of fuel injectors and flameholders in scramjet combustors. These cavities have shown to stabilize hydrocarbon flames at flight Mach numbers between 4 and 6 [21]. An optical bench was set up to enable species measurements in a wall cavity over a supersonic freestream. The laser box and the detector box were mounted on the same plate near the test section to allow ease of traversing the test section in increments of 0.200 " in two directions using translation stages. The cavity region was probed in a $4 \times 11$ grid pattern, 
resulting in 44 measurement locations across the cavity. Stainless steel tubes extend out of the boxes to near $(<1 / 2 "$ away) the tunnel sapphire windows. The boxes are $\mathrm{N}_{2}$-purged, and the purged gases exit these tubes to reduce ambient air in the paths external to the tunnel.

The supersonic air exits a duct (1.5" wide× 1 " high) and passes over a cavity that is 0.6 " deep $\times 2.4$ " long for $\mathrm{L} / \mathrm{D}=4$. Three fuel injectors are located on the wall facing upstream. There are three $1 / 16$ " diameter injectors located 0.2 " above the cavity floor. The side injectors are $0.375 "$ from the walls and the middle one is on the duct centerline. The cavity leading edge is about 4" downstream of the converging-diverging nozzle exit. The Mach number is 2.2 using a ratio of total to static pressure. The total pressure in the non-reacting case is 97.4 psia, so the static pressure in the cavity is 9.1 psia. In the reacting case, the static pressure is 11.6 psia. It is reasonable to assume that the static pressure is approximately constant across the height of the duct. The total temperature of the air for the non-reacting case is 333K, and for the reacting case 533K. The recovery factor in the cavity is about 0.9. So the static temperature in the cavity is $300 \mathrm{~K}$ for the non-reacting case. The estimated flowrates are $0.6 \mathrm{~kg} / \mathrm{s}$ of air, and $0.3 \mathrm{~g} / \mathrm{s}$ of methane, using the assumption of choked nozzle flow.

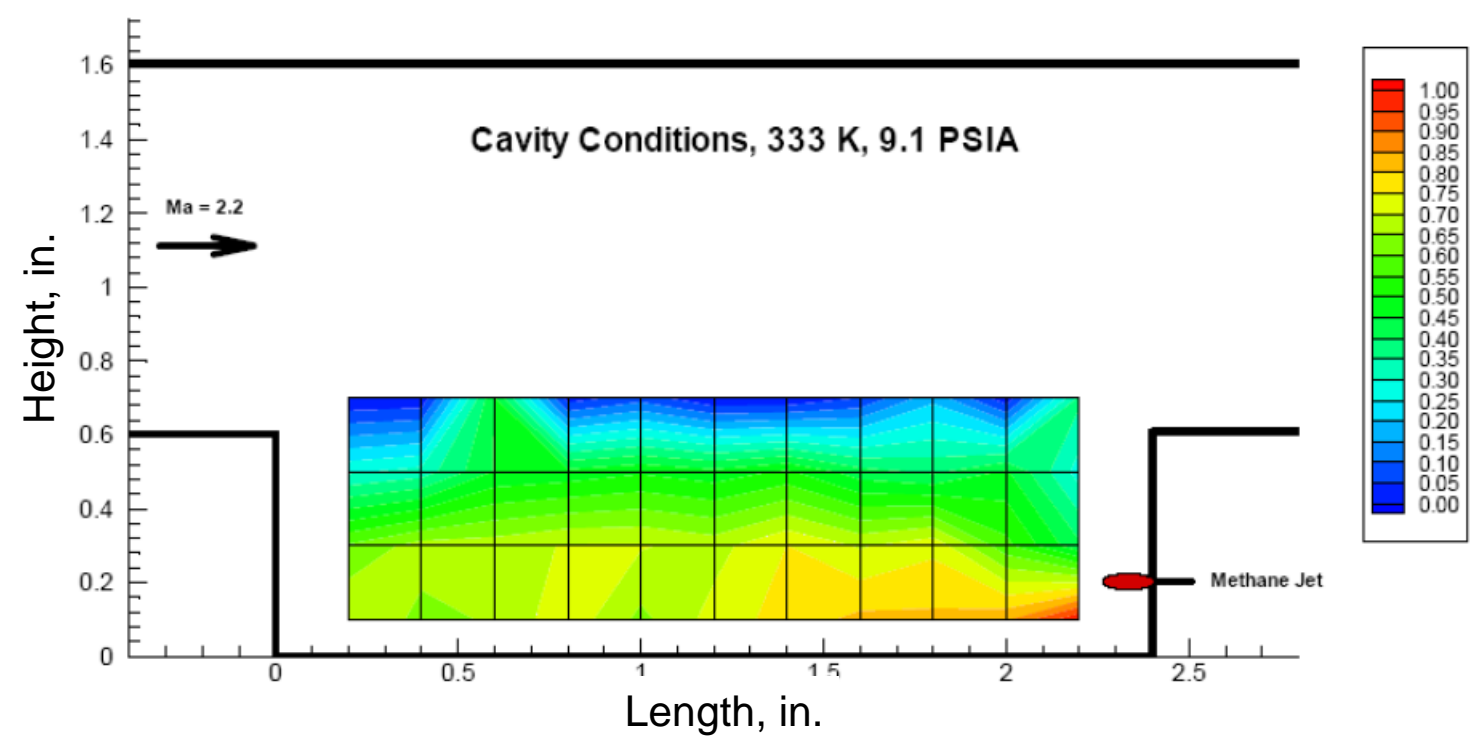

Figure 11. Methane concentrations in a wall cavity over a supersonic freestream.

Measurements in both non-reacting and reacting methane-air cases were initially conducted. The thickness of the sapphire windows (1/2") has generated optical interferences in the $2 f$ absorption signals leading to a reduction in the sensitivity of the detection system. Furthermore, the long external optical paths inside the laser box has contributed large $\mathrm{O}_{2}$ and $\mathrm{H}_{2} \mathrm{O}$ backgrounds that could not be completely removed by $\mathrm{N}_{2}$-purging. Optical interferences were large enough to render $\mathrm{O}_{2}$ and $\mathrm{H}_{2} \mathrm{O}$ measurements inaccurate. The optical path across the cavity is only 1.5 " $(3.81 \mathrm{~cm})$. Given the noise (etalon) in the tunnel due to the thick windows and background absorption, it was not possible to accurately detect $\mathrm{O}_{2}$ concentrations. Extraneous optical paths and optical interferences will be minimized in future tunnel measurements by fiberizing lasers and reducing window thickness. Methane concentrations (in percent volume) in non-reacting cavity flow were measured only. Figure 11 shows higher methane concentrations in the lower portion of the cavity, and low concentrations in the upper region near the shear layer. The clockwise recirculation zone inside the cavity, induced by the supersonic freestream, is responsible for the observed methane distribution.

\section{Summary}

Simultaneous multi-species measurements of chemical species and temperature in a combustion system are demonstrated using modulation-frequency multiplexing to combine several laser wavelengths and a two-color photodiode detector. No channel cross-talk is observed between the Si and InGaAs detector elements of the two-color photodiode detector when the lasers are modulated at the same frequency. Moreover, there is no cross-talk between laser 
wavelengths that are modulated at frequencies separated by more than $1 / 10 \mathrm{kHz}$ and use the same detector element. Wavelength modulation spectroscopy allows high-sensitivity detection of chemical species and the ability to simultaneously detect several laser wavelengths using a single photodiode detector covering the spectral range of interest. Fiberization of the laser sources and utilization of digital signal processors will enable a rugged, compact, stand-alone multi-species sensor system to be designed for flight instrumentation in a scramjet engine.

\section{Acknowledgments}

This project is supported by the Air Force Office of Scientific Research (AFOSR) with contract number FA9550-04C-0102, under the Small Business Technology Transfer (STTR) program, with Dr. Julian Tishkoff as the Program Manager.

\section{References}

1. $\quad$ Baer, D. S., Hanson, R. K., Newfield, M. E., and Gopaul, N. K. J. M. (1994), Opt. Lett. 19:1900-1903.

2. $\quad$ Silver, J. A., D. J. Kane, et al. (1995). Appl. Opt. 34(15): 2787-2801

3. $\quad$ Allen, M. G. (1998), Meas. Sci. Technol. 9(4): 545-562.

4. $\quad$ Mihalcea, R. M., D. S. Baer, and Hanson, R. K. (1998), Proc. Combust. Inst. 27: 95-101.

5. $\quad$ Webber, M. E., J. Wang, Sanders, S. T., Baer, D. S., and Hanson, R. K. (2000), Proc. Combust. Inst. 28: 407-413.

6. $\quad$ S.-J. Chen, J.A. Silver, W.J.A. Dahm, and N.D. Piltch (2002), Proc. Combust. Inst., 29:2679-2685.

7. W.J.A. Dahm, S.-J. Chen, J.A. Silver, J.A. Mullin, and N.D. Piltch (2002), Proc. Combust. Inst. 29:2519-2526

8. $\quad$ Nikkari, J. J., J. M. Di Lorio, and Thomson, M. J. (2002), Appl. Opt. 41(3): 446-452.

9. $\quad$ Arroyo, M. P., Birbeck, T. P., Baer, D. S., and Hanson, R. K. (1994), Opt. Lett. 19:1091-1093.

10. Furlong, E. R., Baer, D. S., and Hanson, R. K. (1998), Proc. Combust. Inst. 27:103-111.

11. Oh, D. B., Paige, M. E., and Bomse, D. S. (1998), Appl. Opt. 37(12), 2499-2501.

12. Silver, J. A., J. Appl. Opt. 31, 707-717 (1992).

13. Bomse, D. S., Stanton, A. C., and Silver, J. A., Appl. Opt. 31, 718-731 (1992).

14. Carlisle, C.B., D.E. Cooper, Appl. Opt. 28, 2567-2576, 1989.

15. Sonnenfroh, D.M., Sewell, S.D., Allen, M.G., in Application of Tunable Diode and Other Infrared Sources for Atmospheric Studies and Industrial Process Monitoring, SPIE Proceedings 2834, 57-66, 1996.

16. Silver, J. A. and Stanton, A. C., Appl. Opt. 27, 4438 (1988).

17. Bomse, D. S., Appl. Opt. 30, 2922 (1991).

18. Stanton, A. C., Silver, J. A., Bomse, D. S., Oh, D. B., Hovde, D. C., Paige, M. E., and Kane, D. J., in Application of Tunable Diode and Other Infrared Sources for Atmospheric Studies and Industrial Process Monitoring, A. Fried, ed., Proc. SPIE 2834, 41 (1996).

19. Silver, J. A. and Hovde, D. C., Rev. Sci. Instrum. 65, 1691-1694 (1994).

20. Hovde, D. C., Meyers, T. P., Stanton, A. C. and Matt, D. R., J. Atmos. Chem. 20, 141 (1995).

21. Mathur, T., et al., J. Prop. Power 17 (2001). 